Archives

4| 1989

Varia

\title{
Techniques d'irrigation et société hydraulique à Ceylan à la période pré-coloniale
}

\section{Eric Meyer}

\section{(2) OpenEdition}

\section{Journals}

Édition électronique

URL : http://journals.openedition.org/ccrh/2902

DOI : $10.4000 /$ ccrh.2902

ISSN : 1760-7906

Éditeur

Centre de recherches historiques - EHESS

Édition imprimée

Date de publication : 15 octobre 1989

ISSN : 0990-9141

Référence électronique

Eric Meyer, «Techniques d'irrigation et société hydraulique à Ceylan à la période pré-coloniale », Les Cahiers du Centre de Recherches Historiques [En ligne], 4 | 1989, mis en ligne le 13 avril 2009, consulté le 19 avril 2019. URL : http://journals.openedition.org/ccrh/2902 ; DOI : 10.4000/ccrh.2902

Ce document a été généré automatiquement le 19 avril 2019

Article L.111-1 du Code de la propriété intellectuelle. 


\title{
Techniques d'irrigation et société hydraulique à Ceylan à la période pré- coloniale
}

\author{
Eric Meyer
}

1 Cette intervention vise à fournir des éléments au débat sur la relation entre les techniques d'irrigation et l'organisation socio-politique qualifiée d'hydraulique, à partir d'une étude de cas limitée à Ceylan, et accessoirement à l'Inde du Sud.

2 Alors que Ceylan offre l'exemple le plus achevé en Asie du Sud d'une économie hydraulique très élaborée, il est singulier que Wittfogel, dans son ouvrage classique sur le

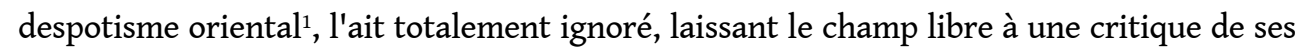
thèses, qui, dans ce cas exemplaire, ne résistent guère à l'examen. Plusieurs anthropologues et historiens spécialistes de Ceylan se sont livrés à une critique dévastatrice du modèle Wittfogélien : nous retiendrons ici les travaux de Leach ${ }^{2}$ et ceux de Gunawardana ${ }^{3}$. Leach, avec son génie habituel pour le paradoxe, inverse les assertions de Wittfogel et soutient que l'économie hydraulique est produite par une société qu'il qualifie de féodale. L'analyse de l'historien ceylanais Gunawardana, plus approfondie et à bien des égards plus convaincante, met l'accent sur les rapports centre-périphérie dans le système hydraulique, et souligne le rôle respectif de la monarchie et des monastères bouddhiques dans la genèse et l'entretien du système.

3 J'insisterai ici sur les aspects techniques de l'hydraulique ${ }^{4}$ : pour en comprendre les impératifs, il convient de rappeler les grands traits du milieu naturel de l'île, et de l'histoire de sa mise en valeur.

\section{La nature et l'histoire}

4 Le relief et les conditions climatiques prévalant dans l'île individualisent deux espaces biogéographiques très contrastés: le centre montagneux et le quart sud-Ouest bénéficient de précipitations régulières réparties sur l'ensemble de l'année : alors que le 
Nord et l'Est de l'île sont arrosés durant deux ou trois mois seulement, et avec des risques de fort déficit pluviométrique d'une année à l'autre.

5 Ce contraste s'explique par la présence du massif montagneux central qui reçoit les pluies abondantes apportées par la mousson humide du Sud-Ouest de mai à septembre, l'atmosphère étant ensuite assez saturée pour que des pluies de convection soient assurées durant le reste de l'année : situation comparable à celle du Kerala (Sud-Ouest de l'Inde). Au contraire le reste de l'île a un régime climatique identique à celui du Tamilnadu (Sud-Est de l'Inde) : la zone montagneuse fait écran à la mousson du SudOuest, et les pluies ne surviennent que lorsque souffle la mousson du Nord-Est (novembre-janvier) qui se charge d'un peu d'humidité en traversant le Golfe du Bengale.

6 Les fleuves qui prennent naissance dans le massif montagneux sont régulièrement alimentés; l'un d'entre eux, le Mahaveli Ganga (ainsi que son affluent l'Amban Ganga), coule en direction de la zone sèche; mais la plupart des cours d'eau du Nord et de l'Est sont taris une partie de l'année; la péninsule de Jaffna, qui bénéficie d'une nappe phréatique bien alimentée, fait exception. La végétation naturelle est partout dense, mais sa croissance est beaucoup plus rapide dans la zone humide, dont les sols sont souvent lourds et marécageux, et dont la faune (éléphants sauvages notamment) est plus difficile à maîtriser.

7 On peut distinguer dans l'histoire de l'aménagement agricole de l'île trois phases qui correspondent à trois états des techniques d'irrigation et d'essartage : entre le $\mathrm{v}^{\mathrm{e}}$ siècle avant notre ère et le XII ${ }^{\mathrm{e}}$ siècle, $c^{\prime}$ est la zone sèche qui est progressivement mise en valeur, grâce à un système hydraulique de plus en plus élaboré, qui fait l'objet de la présente étude; à partir du $\mathrm{XIII}^{\mathrm{e}}$ siècle, cette civilisation hydraulique est victime d'une crise profonde, et la quasi-totalité de la population se replie dans la zone humide (et accessoirement à Jaffna) qu'elle exploite dans un système de polyculture où l'irrigation occupe une place bien moindre. Ce n'est qu'à la fin du XIX siècle que certains ouvrages hydrauliques sont restaurés dans la zone sèche, et son repeuplement n'intervient réellement qu'au cours $\mathrm{du} \mathrm{xx}^{\mathrm{e}}$ siècle. Cette histoire nous est connue dans ses grandes lignes grâce à l'existence d'une chronique continue depuis le III ${ }^{\mathrm{e}}$ siècle avant notre ère, le Mahavamsa, dont les indications sont complétées par des inscriptions surtout abondantes à partir du vIII ${ }^{\mathrm{e}}$ siècle, et sur le plan technique qui nous intéresse ici, par les données particulièrement précises fournies par l'archéologie et les levés topographiques.

8 La riziculture irriguée semble avoir été introduite dans l'île depuis l'Inde proche vers le ve siècle avant notre ère. La zone sèche, plus facile à essarter que la zone humide, bénéficiant d'un meilleur ensoleillement, était plus propice à la riziculture sur le modèle indien que la zone humide, qui resta occupée par une jungle épaisse. Il est probable que les populations antérieurement établies se livraient à la chasse et peut-être à la culture sèche sur brûlis. Jusqu'aux débuts de notre ère, les réservoirs villageois, dont le modèle est identique à ceux qu'on trouve au Tamilnadu, ne permettent de conserver les eaux de la mousson du Nord-Est que pour la durée d'une saison de culture, sans pouvoir conjurer les risques de mousson déficiente : des cas de famine sont fréquemment signalés.

9 A partir du $\mathrm{I}^{\mathrm{er}}$ siècle (règne de Vasabha. 67-111), des canaux de dérivation permettent de capter les eaux des fleuves descendus des montagnes. Les premiers réservoirs de vaste dimension datent du $\mathrm{III}^{\mathrm{e}}$ siècle (Mahasena, 274-301), puis des réseaux complexes reliant les réservoirs les uns aux autres sont peu à peu constitués entre le $\mathrm{v}^{\mathrm{e}}$ et le $\mathrm{VII}^{\mathrm{e}}$ siècle. 
Après une phase de dégradation passagère, le système connait son extension maximale sous le règne de Parakramabahu 1er (1153-1186).

Le déclin du système intervient dès le XIII ${ }^{\mathrm{e}}$ siècle. Le phénomène a fait l'objet de nombreuses études ${ }^{5}$, sans que ses causes aient pu être bien élucidées : il semble qu'il y ait eu convergence de facteurs externes (destructions causées par des invasions Sudindiennes, possible dégradation climatique) et de facteurs internes (entropie du système s'accompagnant d'une crise socio-politique); le glissement de la population vers la zone humide a certainement répondu en outre à des possibilités nouvelles ouvertes par de meilleurs outils et armes de fer, des techniques d'abattage et de drainage plus efficaces, l'amélioration des espèces végétales (cocotiers et différentes variétés de palmiers), et l'attirance du commerce maritime; enfin, l'extension du paludisme sur les sites hydrauliques abandonnés en a rendu impossible la recolonisation jusqu'à l'utilisation du DDT dans la lutte contre l'anophèle.

\section{Techniques d'irrigation et milieu naturel}

11 On s'interrogera ici sur le choix, la genèse et la transmission des techniques d'irrigation, et sur leur combinaison pour former des systèmes hydrauliques, à partir d'un modèle logique documenté par les sources archéologiques et topographiques ${ }^{6}$. Il s'agit là d'une reconstitution hypothétique des grandes tendances de l'évolution, qui ne rend pas nécessairement compte de situations locales particulières.

Les premiers riziculteurs s'établirent dans les plaines sèches du Nord, barrant de légers vallonnements pour former des étangs de petites dimensions ( 1 à $2 \mathrm{ha}$ ) destinés à irriguer les terres d'un seul village. Ce type d'ouvrage hydraulique s'est perpétué jusqu'à nos jours, survivant au déclin des grands systèmes hydrauliques ${ }^{7}$. Mais il ne permettait pas de conserver l'eau pendant plus d'une saison de culture, ni de conjurer les risques de famine en cas de mousson déficiente. Cette petite irrigation était incapable de dégager des surplus ; elle était en quelque sorte répartitrice, distribuant la pénurie ou l'abondance, et s'accompagnait d'une organisation sociale relativement égalitaire. Elle ne pouvait se développer pour accompagner une éventuelle croissance de la population que par duplication du modèle initial : et les levés topographiques ont permis de retrouver dans certaines régions les traces de centaines d'étangs identiques, signes d'une densification sans développement. Au bout d'un certain temps, les sites adaptés étaient tous occupés, le modèle initial entra en crise et il fallut trouver des solutions nouvelles.

L'irrigation en réseaux qui apparait au cours $d u \mathrm{I}^{\mathrm{er}}$ siècle de notre ère représente un changement d'échelle et résulte d'une mutation en matière d'organisation de la production. D'une étendue initiale d'une centaine d'hectares, les lacs artificiels atteignent 3.000 à 4.000 hectares au $\mathrm{VI}^{\mathrm{e}}$ siècle : les plus grands permettent d'irriguer 40.000 hectares. L'eau est retenue par des digues qui peuvent avoir jusqu'à $14 \mathrm{~km}$ de long, $23 \mathrm{~m}$ de haut. Les canaux d'amenée ou de décharge, de pente très faible (1 pour 10.000), ont pour certains $30 \mathrm{~m}$ de large et $80 \mathrm{~km}$ de long. On n'hésite pas à dériver l'eau des rivières de montagne les plus puissantes en établissant des chaussées (anicut) au travers du courant. Ce changement d'échelle implique la maîtrise de techniques nouvelles d'une part, et l'aptitude des bâtisseurs à mobiliser une importante main d'oeuvre durant une longue période. On reviendra sur ces points plus loin. 
14 Mais le nouveau système implique aussi un changement d'organisation, une volonté de planification fondée sur l'idée d'optimisation de l'usage de l'eau (qu'exprime d'après la chronique le roi du XII ${ }^{\mathrm{e}}$ siècle Parakramabahu $\mathrm{I}^{\mathrm{er}}$ lorsqu'il édicte que pas une goutte d'eau ne doit atteindre la mer sans avoir servi à fertiliser son royaume), et de régularisation de l'approvisionnement, qui permet dès lors deux récoltes annuelles de paddy, quels que soient les aléas climatiques. L'idée de développement se manifeste à travers une volonté de perfectionnement constant du système, qui ne cesse d'évoluer au cours des dix siècles de son existence. Il s'agit bien là, pour reprendre la terminologie de Wittfogel, d'une irrigation « créatrice ».

Ces principes sont mis en oeuvre d'abord en alimentant les réservoirs à partir des rivières pérennes issues de la zone humide, que l'on dérive à la sortie des montagnes; en même temps on s'efforce de diversifier les sources d'approvisionnement en eau de chaque réservoir ; le cas échéant, on fait passer l'eau d'un bassin dans un autre, ou on la conduit dans une dépression naturelle où il est plus facile d'édifier une digue que dans la vallée principale. Enfin, on relie les réservoirs les uns aux autres pour former plusieurs grands réseaux en chapelets, longs chacun d'une centaine de kilomètres; ceux du Nord-Ouest dépendent du grand lac de Kalawewa, alimenté partiellement par la capture des eaux de l'Amban Ganga et irriguent les deux vallées du Kala Oya et du Malwattu Oya (où est située la première capitale Anuradhapura); ceux du Nord-Est captent les eaux du Mahaveli Ganga et de ses affluents et les conduisent jusqu'à la mer en irriguant toutes les terres de rive gauche, où se trouve la seconde capitale Polonnaruwa.

Pour rendre possible cette révolution hydraulique, un certain nombre d'obstacles techniques très spécifiques ont dû être surmontés à l'aide de procédés dont le perfectionnement semble résulter d'une expérimentation par tâtonnement (by trial and error selon l'excellente expression anglaise).

Parmi les techniques mises en oeuvre, il faut souligner que certaines impliquent des connaissances de planimétrie et de nivellement; ainsi le tracé des canaux qui épousent les courbes de niveau de façon à perdre le moins possible d'altitude ; ou la pratique qui consiste à entreprendre simultanément en plusieurs points la construction d'un ouvrage hydraulique.

18 Le principal problème était le risque de brèche par érosion des digues, que mentionnent de nombreux témoignages (et que les habitants ont cherché à conjurer par des rites et des pratiques tels que des sacrifices d'animaux ou d'humains enterrés dans le barrage, dont il restait quelques survivances au début du XIX ${ }^{e}$ siècle). Les points faibles de la digue étaient le déversoir permettant d'évacuer les crues, la face interne du réservoir exposée aux vagues en périodes de grand vent, et surtout les chenaux d'irrigation traversant la base de la digue. On multiplia les déversoirs et on les revêtit de pierres appareillées. On arma l'âme des digues avec des blocs de pierre, on en compacta la terre en employant sans doute des éléphants, et on la munit sur sa face interne de parements de pierres orientées de biais pour servir de brise-vagues (relapanawa).

19 L'effort majeur porta sur les chenaux d'écoulement ${ }^{8}$. Dans les petits étangs villageois, la sortie de l'eau se faisait par de simples tranchées comblées en fin de saison ; les réservoirs plus vastes devaient avoir des vannes à débit réglable, mais les tunnels édifiés sous la base de la digue, si bien appareillés fussent-ils, risquaient de s'effondrer ou d'être obstrués par des débris. Les hydrauliciens mirent au point un procédé complexe de vanne-puits bisokotuwa) permettant de régler le débit de l'eau en dépit de très fortes pressions 
initiales, et de réduire progressivement cette pression au passage sous la base de la digue : l'eau était menée au fond d'un puits de section rectangulaire édifié en bordure intérieure de la digue, par une conduite de pierre immergée y pénétrant en oblique; la sortie de l'eau se faisait à un niveau plus élevé par une seconde conduite munie d'une vanne à son entrée, enduite d'un ciment à base d'argile pour éviter les infiltrations, et s'élargissant progressivement jusqu'à son débouché à l'extérieur de la digue, de façon à jouer le rôle de réducteur de pression. Le système était si efficace qu'il a été simplement restauré lors des opérations de reconstruction menées par les Britanniques à partir de la fin du XIXe siècle.

On ignore les origines de cette innovation dont les premiers exemples datent du $\mathrm{III}^{\mathrm{e}}$ siècle, et sont donc antérieurs à l'édification des plus grands barrages qu'elle a rendue possible. Il existe des procédés obéissant au même principe mais beaucoup plus sommaires dans l'Espagne romaine, et des systèmes complexes de vannes sur les canaux chinois, mais qui répondent à des impératifs techniques tout à fait différents ${ }^{9}$. Quant à l'hydraulique Sudindienne, qui se développe dans la vallée de la Kaveri vers la même époque, avec le premier grand barrage situé à Tanjore et que l'on date du milieu du II ${ }^{\mathrm{e}}$ siècle, elle ne semble avoir adopté que plus tard et à plus petite échelle ces techniques sophistiquées. Le développement du système d'irrigation dans l'ensemble du Tamilnad est postérieur au $\mathrm{VIII}^{\mathrm{e}}$ siècle, et il faut attendre le $\mathrm{XII}^{\mathrm{e}}$ siècle pour que s'y édifie un réseau complexe de canaux et de réservoirs comparable à celui de Ceylan, au moment même où ce système entre en déclin dans l'île ${ }^{10}$.

21 Si un processus de transmission des techniques a eu lieu, il est donc probable qu'il s'est fait dans le sens Ceylan-Inde, les innovations techniques les plus décisives étant apparues en vertu d'une évolution endogène, que Needham, le seul spécialiste à offrir une vue comparative de la question, estime fort possible, à la différence des techniques métallurgiques ou de construction navales caractérisées selon lui par des transferts à longue distance. Mais il ne faut pas négliger l'apport initial de techniques simples, qu'il s'agisse de la riziculture irriguée d'origine extrême-orientale, ou de procédés d'irrigation employés dans les cités de l'Indus et au Proche-Orient. Il reste enfin à s'interroger sur les groupes sociaux qui étaient porteurs de ces techniques, et sur les pouvoirs capables d'en organiser la mise en oeuvre.

\section{Hydraulique et société}

22 Avant de reprendre les éléments du débat soulevé par les thèses de Wittfogel, à la lumière de ce qui vient d'être dit des techniques hydrauliques, il faut souligner que ce système n'a pu se développer qu'à certaines conditions :

23 - pour qu'une planification cohérente puisse se poursuivre à très long terme sur des espaces relativement vastes, il faut une continuité et une territorialité du pouvoir, et un intérêt soutenu porté par ce pouvoir à l'activité agricole : toutes choses qui ne sont pas habituelles dans le monde indien, alors qu'elles semblent être la norme en Chine et au Proche-Orient ;

24 - la dimension des ouvrages suppose un investissement-travail considérable, fondé sur l'aptitude d'une autorité à mobiliser la main d'oeuvre, à accumuler des surplus, et à en organiser la répartition; toutefois cet investissement a pu être étalé sur de longues périodes et ne comporte pas nécessairement la présence permanente d'une grande concentration de travailleurs ; 
Gunawardana à la recherche des acteurs du développement hydraulique ${ }^{11}$. L'hypothèse qui se présente en premier lieu est celle d'une monarchie bâtisseuse : Ceylan diffère de la plupart des régions de l'Inde a cet égard; des rois ont constitué autour d'Anuradhapura, à partir du III e siècle avant notre ère, un Etat qui a fini par inclure au cours du premier millénaire de notre ère l'ensemble de l'île; au sein de ce territoire, ils disposaient d'un domaine propre comportant les terroirs les mieux irrigués, et des sources tardives montrent qu'ils pouvaient disposer des services gratuits des habitants (rajakariya), dans le cadre du système des castes que la monarchie avait exploité à ses propres fins ; des textes ceylanais et Sud-indiens mentionnent la présence de castes de tailleurs de pierres affectés à la construction de barrages, et il est possible que le savoir technique se soit transmis au sein de ces groupes comme chez les maçons du Moyen Age occidental, sans que se soit dégagée à proprement parler une classe d'ingénieurs ou d'architectes. Il est en tout cas vraisemblable que travailleurs et techniciens étaient en forte demande, comme l'atteste l'épisode rapporté dans des chroniques tardives du roi d'Anuradhapura, Gajabahu 1er, auquel le roi de Tanjore (Tamilnad) aurait enlevé les hommes qu'il employait pour construire des barrages, et qui aurait organisé une grande expédition pour les ramener d'Inde du Sud et enlevé à son tour par mesure de rétorsion un nombre égal de travailleurs tamouls ${ }^{12}$.

Mais à l'époque où ont été entrepris les ouvrages les plus importants, le processus de centralisation était loin d'être avancé, le roi n'était pas maître de l'ensemble du sol (les inscriptions mentionnent de nombreux cas d'appropriation privée de réservoirs), et il est souvent fait état de salaires que le roi versait à des travailleurs libres à son service ; il n'y a aucun signe que le système hydraulique ait été le produit d'un Etat despotique et bureaucratique. Quant aux traits d'absolutisme qui apparaissent après le $\mathrm{xI}^{\mathrm{e}}$ siècle, ils sont contemporains du déclin du système; en outre, ils se combinent à un régime d'amodiation de la terre en échange de services spécifiques à chaque caste, que Leach qualifie hâtivement à mon sens de féodal (il va jusqu'à forger pour le décrire les concepts hybrides de "féodalisme de caste» et de "féodalisme hydraulique »). En tout état de cause, cette formation socio-politique, caractéristique du royaume de Kandy qui s'est établi dans la zone humide après le $\mathrm{XVI}^{\mathrm{e}}$ siècle, est sans lien aucun avec l'économie hydraulique des royaumes de l'Antiquité.

Le modèle nuancé que propose Gunawardana me semble beaucoup plus convaincant. Il distingue dans le temps et dans l'espace plusieurs sous-systèmes dont l'agencement obéit à une relation centre-périphérie Après une phase initiale où l'initiative privée semble avoir prédominé, l'entreprise des grands ouvrages revient généralement à la monarchie dans les zones centrales, et à des potentats locaux dans les zones périphériques. Mais par le jeu de donations effectuées au profit de monastères bouddhiques ou de grands officiers de la cour, la monarchie a périodiquement aliéné ses prérogatives sur une partie des terroirs irrigués. Dans le cas du Tamilnad étudié par Ludden, où la centralisation monarchique est pratiquement absente (l'historien Burton Stein applique à l'Inde du Sud médiévale le modèle de l'Etat segmentaire), l'initiative locale est seule en jeu, mais catalysée par des mouvements de donation à des temples hindouistes. Cette 
caractéristique nous ramène à un autre volet de l'analyse de Gunawardana, qui mérite à mon sens d'être systématisé.

Il s'agit du rôle joué par les grands monastères bouddhiques dans l'élaboration, et surtout la gestion du système hydraulique. Ils répondent mieux que tout autre organisme aux impératifs posés plus haut comme favorables au développement hydraulique. La continuité de l'autorité y est mieux assurée qu'au palais agité de complots et de luttes de succession. Le territoire contrôlé n'est ni aliénable, ni partageable entre des héritiers, et peut s'accroître de donations nouvelles; les conditions d'accumulation d'un capital foncier et de surplus sont réunies. Quant à la mobilisation de la main d'oeuvre, elle est facilitée par l'attirance qu'exercent les monastères, par les services de caractère rituel que les paysans des villages dépendant d'eux doivent rendre, en échange de la protection qui leur est accordée, et de l'exemption de toute charge au profit de quelque autre autorité; en outre l'effort demandé est valorisé religieusement, l'aumône à la communauté des moines étant considérée comme l'acte méritoire par excellence: les facteurs idéologiques jouent un rôle mobilisateur décisif.

Encore faut-il que l'on choisisse de consacrer les surplus à la construction de barrages plutôt ou au même titre qu'à l'édification de monuments religieux (tels les immenses stupa des capitales antiques) ou qu'à la consommation de moines de plus en plus nombreux et à qui le travail manuel est interdit (à la différence des moines cisterciens par exemple). A ce niveau intervient le fait que les monastères sont des lieux de culture et de science, où la conservation du savoir et des techniques est valorisé, où règne la culture de l'écrit, et où très probablement les ingénieurs de l'Antiquité ont trouvé appui et encouragement. Ce sont des lieux d'échange, que visitent sans cesse des moines originaires de toute l'Asie (les moines de Ceylan sont les dépositaires de la tradition orthodoxe et de ses textes de référence), ce qui peut contribuer à la diffusion de techniques nouvelles et plus généralement développer l'inventivité. Ce sont enfin des lieux où les pratiques de comptabilité sont répandues, comme en témoignent les archives d'un monastère du IXe siècle (tablettes de Mihintale) : le calcul économique fait partie de la culture monastique ; la rationalité économique n'est pas consciemment affirmée, mais sa logique est déjà présente. Max Weber a bien souligné ce "paradoxe de tout ascétisme rationnel, qui crée la richesse qu'il condamne : partout les temples et les monastères sont devenus les lieux privilégiés de toute économie rationnelle $»^{13}$.

\section{NOTES}

1. Karl WITTFOGEL, Oriental Despotism, Newhaven (Connecticut) 1957, trad. française. Le despotisme oriental, Paris, éd. de Minuit, 1964.

2. Edmund LEACH, « Hydraulic Society in Ceylon ", Past and Present, $n^{\circ} 15$ (1959) pp. 2-26.

3. R.A.L.H. GUNAWARDANA, 1971, «Irrigation and Hydraulic Society in Early Mediaeval Ceylon », Past and Present, 53 (1971), pp. 2-27, Id. 1978a, " Social Function and Political Power : a Case Study in State Formation in Irrigation Society", Indian Historical Review, IV 2 (1978), pp. 259-273. 
4. Pour une étude de la nature de la société antique, voir Eric MEYER, « Comment caractériser les royaumes sri-lankais anciens? Note critique à propos de quelques travaux récents, » Purushartha XIII (1990). L'État dans le monde indien : Histoire et Sociologie.

5. K. INDRAPALA (ed), The Collapse of the Rajarata Civilization in Ceylon and the Drift to the South West, a Symposium. Peradeniya, University of Ceylon, 1971.

6. Rassemblées par R.L. BROHIER, Ancient Irrigation Works in Ceylon, Colombo, Government, Press, 1934,3 vol.

7. Une description détaillée en est donnée par E.R. LEACH, Pul Eliya, a Village in Ceylon, Cambridge, 1961.

8. R.A.L.H. GUNAWARDANA, « Hydraulic Engineering in Ancient Sri Lanka : the Cistern Sluices », Senarat Paranavitana Commemoration Voume, Leiden. Brill. 1978, pp. 61-74.

9. J. NEEDHAM, Science and Civilization in China, vol. IV, part 3, Cambridge, 1971, p. 367 sq.

10. E. ADICEAM, La géographie de l'irrigation dans le Tamilnad. Paris, École Française d'Extrême Orient, 1966, J. LUDDEN, «Patronage and Irrigation in Tamilnad, a Long Term View ». Indian Economic and Social History Review, XVI, 3 (1979).

11. Outre les travaux de ces auteurs, ainsi que l'article de LUDDEN, cités ci-dessus, voir R.A.L.H. GUNAWARDANA, Robe and Plough: Monasticism and Economic Interest in Early Mediaeval Sri Lanka, Tucson University of Arizona Press. 1979, ainsi que K.M. de SILVA, A History of Sri Lanka, Londres \& Berkeley, Hurst. 1981.

12. L'épisode a été interprété comme un mythe visant à expliquer la présence d'une population d'origine tamoule à Ceylan (cf G. OBEYESEKERE, «Gajabahu and the Gajabahu Synchronism, an inquiry into the relationship between myth and history " in Religion and the Legitimation of Power in Sri Lanka. B.L. Smith, ed. Chambersburg, Anima Books, 1978); mais le récit comporte certainement un fonds de vérité, même si sa datation est impossible.

13. Max WEBER, «Religious Rejections of the World and their Directions » in From Max Weber, H.H. Gerth \& C. Wright Mills, ed, London, 1961, p. 332.

\section{AUTEUR}

\section{ERIC MEYER}

Eric MEYER est directeur du Centre de l'Inde et de l'Asie du Sud (EHESS/CNRS). 